\title{
Dermatology Residency Applicants' Concerns Due to COVID-19
}

\author{
Kayla A. Clark, BS ${ }^{1}$, Virginia M. Miori, $\mathrm{PhD}^{2}$, Virginia A. Jones, MD, MS ${ }^{1}$, Maria M. Tsoukas, \\ $\mathrm{MD}, \mathrm{PhD}^{1}$ \\ ${ }^{1}$ Department of Dermatology, University of Illinois at Chicago, Chicago, IL \\ ${ }^{2}$ Department of Decision and System Sciences, Saint Joseph's University, Philadelphia, PA
}

\section{ABSTRACT}

Background: The COVID-19 pandemic caused substantial disruptions to medical education. We hypothesized that these disruptions may affect students applying to competitive residencies such as dermatology.

Methods: A 24-question IRB-exempt, online, anonymous survey was distributed to 240 eligible medical students via social media to assess dermatology residency applicants' concerns due to the COVID-19 pandemic and to collect student-proposed solutions to assist with the virtual application cycle. Eligibility consisted of US MD students applying to dermatology residency in the 2020-2021 or 2021-2022 application cycles. Statistical significance was calculated using the Chi-squared procedure, Ttest/ANOVA, Mann Whitney test, and Kruskal-Wallis tests with post-hoc Tukey and Mann Whitney tests.

Results: Of 240 eligible students, 77 students attempted the survey whereas 69 students completed it (response rate $32 \%$, completion rate $89 \%$ ). Salient findings include students without home dermatology departments (WHD; $n=24)$ who demonstrated concerns about research project changes $(p=0.00)$. Students under-represented in medicine (UIM; $n=34)$ concerned about lack of in-person interviews $(p=0.00 ; p=0.04)$. MS3 $(n=14)$, concerned about test cost $(p=0.00)$, and effect on test scores because of COVID-19 $(p=0.03)$. WHD and UIM applicants showed concerns about changes in clinical experiences $(p=0.03, p=0.03$, respectively). Lastly, WHD, UIM and MS3 expressed concerns about obtaining quality recommendation letters $(p=0.00, p=0.04$, and $p=0.03$, respectively).

Conclusion: Our findings demonstrate the various concerns shared by dermatology residency applicants because of the COVID-19 pandemic. The suggested solutions offered by participants in our study can be used to improve processes for candidates in the 2021-2022 residency application cycle.

Educational policy changes related to the Coronavirus disease 2019 (COVID-19) pandemic during the 2020-2021 residency application cycle caused challenges for dermatology residency applicants. ${ }^{1}$ As advised by the Association of American Medical Colleges (AAMC), programs conducted interviews entirely virtually. ${ }^{2}$ Due to these unique circumstances, our group created a survey to gain insight into dermatology residency applicants' concerns attributable to the COVID-19 pandemic and to gauge which solutions they proposed.

A 24-item, IRB-exempt, anonymous, online survey, was distributed to 240 medical students via social media (GroupMe, Twitter, and Instagram). Eligibility was limited to US MD students applying to dermatology residency in the $2020-2021$ or 2021-2022 
application cycles. Responses were collected over a two-week period. Data analysis was conducted using software (SPSS, Version 27, IBM Corp, Armonk, NY).

Numerical values were generated for (1) students with and without home dermatology departments (WHD), (2) students who selfidentified as underrepresented in medicine (UIM) and non-UIM, and (3) medical school class (MS3, MS4 and Other) with "No Concerns" and "Extremely Concerned" valued at 1 and 5 , respectively. Statistical significance was calculated using the Chisquared procedure, T-test/ANOVA, Mann Whitney test, and Kruskil Wallis tests with post-hoc Tukey and Mann Whitney tests.

Of 240 eligible students, 77 students attempted the survey whereas 69 students completed it (response rate (RR) $32 \%$, completion rate $89 \%$ ). Statistically significant survey responses and respondent demographics are summarized in Table 1 and Table 2, respectively. Salient findings include WHD $(n=24)$, demonstrated concern about research project changes $(p=0.00)$. UIM ( $n=34)$, had concerns about lack of inperson interviews $(p=0.00 ; p=0.04)$. MS3 $(n=14)$, concerns about test cost $(p=0.00)$ and believed test scores may be affected by COVID-19 $(p=0.03)$. WHD and UIM applicants showed concerns about changes in clinical experiences $(p=0.03, p=0.03$, respectively). WHD, UIM and MS3 showed concerns about obtaining high-quality letters of recommendation (LOR) $(p=0.00, p=0.04$, and $p=0.03$, respectively).

Our findings demonstrate that students had significant concerns regarding the 2020-2021 and 2021-2022 application cycles (Table 1). Those directly related to the virtual match include concerns of accurate sense of program culture, concerns with acquisition of LORs, and concerns of lack of interaction with fellow interviewees. Despite these concerns, preliminary data released by the AAMC reveals an $8 \%$ (661 students vs. 611) increase for MD students applying to dermatology residency this cycle. ${ }^{3}$ Preliminary match results indicate a $73.9 \%$ (382/517) match rate for PGY-2 position for MD seniors in the 2020-2021 cycle compared to the $78.1 \%(368 / 471)$ match rate in the 2019-2020 cycle; an overall $4.2 \%$ decrease in the match rate. 4,5 Consistent with MD applicant concerns, this cycle's virtual match yielded a lower match rate. We believe the student-proposed solutions from our study may serve to mitigate anxiety accompanied with the use of virtual interviews in the upcoming cycle. These solutions include virtual meetings with program leadership and residents, which allow for exposure to the programs culture and virtual mentorship which affords the opportunity to acquire a LOR. All student-proposed solutions are summarized in Figure 1. Furthermore, we believe that interviewed applicants may benefit from optional in-person second look events to gain more insight into the program culture and to interact with fellow candidates. However, programs would have to ensure that this event would have not influence their rank order list.

Our study is limited by a small cohort size, low RR, and a narrow demographic. However, we hope our proposed solutions will allow programs to adjust for applicants' concerns in the upcoming cycle. In addition, we continue to advocate for the use of holistic review, emphasis on "distance traveled", and that programs strive for residency classes that mirror the diverse demographics of the United States. 


\section{SKIN}

Table 1. Statistically Significant Survey Responses

\begin{tabular}{|c|c|c|c|c|}
\hline & $\begin{array}{c}\text { Category } \\
\text { (n, mean, median) }\end{array}$ & Response $(\mathrm{n} 1, \mathrm{n} 2, \mathrm{n} 3)$ & $\begin{array}{l}P \\
\text { value }\end{array}$ & $\begin{array}{l}\text { Significant paired } \\
\text { comparison ( } p \\
\text { value) }\end{array}$ \\
\hline \multirow[t]{2}{*}{$\begin{array}{l}\text { How concerned are you about your } \\
\text { USMLE STEP } 1 \text { or USMLE STEP } 2 \text { CK } \\
\text { scores being affected by the COVID-19 } \\
\text { pandemic? }\end{array}$} & $\begin{array}{l}\text { UIM }(34,2.82,3.00)^{€} \\
\text { Non-UIM }(33,2.09,2.00)\end{array}$ & $\begin{array}{l}\text { No concern }(10,16) \\
\text { Slightly }(3,6) \\
\text { Somewhat }(11,5) \\
\text { Moderately }(3,4) \\
\text { Extremely }(7,2)\end{array}$ & $0.04^{t}$ & \\
\hline & $\begin{array}{l}\text { MS3 }(14,3.36,3.00) \\
\text { MS4 }(50,2.22,2.00) \\
\text { Other }(5,2.00,2.00)\end{array}$ & $\begin{array}{l}\text { No concern }(2,24,1) \\
\text { Slightly }(0,7,3) \\
\text { Somewhat }(7,8,1) \\
\text { Moderately }(1,6,0) \\
\text { Extremely }(4,5,0)\end{array}$ & $0.02^{1}$ & $\begin{array}{l}\text { MS3-MS4 }(0.01)^{t} \\
\text { MS3-Other }(0.03)^{ \pm}\end{array}$ \\
\hline \multirow[t]{2}{*}{$\begin{array}{l}\text { How concerned are you about changes in } \\
\text { your dermatological clinical } \\
\text { experience(s)? }\end{array}$} & $\begin{array}{l}\text { UIM }(34,3.74,4.00)^{€} \\
\text { Non-UIM }(33,3.09,3.00)\end{array}$ & $\begin{array}{l}\text { No concern }(1,4) \\
\text { Slightly }(, 5) \\
\text { Somewhat }(6,13) \\
\text { Moderately }(9,6) \\
\text { Extremely }(12,5)\end{array}$ & $0.03^{t}$ & \\
\hline & $\begin{array}{l}\text { Home Derm Dept }(45,3.18,3.00) \\
\text { Not Home Derm Dept }(24,3.88,4.00)\end{array}$ & $\begin{array}{l}\text { No concern }(5,0) \\
\text { Slightly }(10,2) \\
\text { Somewhat }(13,6) \\
\text { Moderately }(6,9) \\
\text { Extremely }(11,7)\end{array}$ & $0.03^{t}$ & \\
\hline $\begin{array}{l}\text { How concerned are you about changes to } \\
\text { your research projects? }\end{array}$ & $\begin{array}{l}\text { Home Derm Dept }(45,2.91,3.00) \\
\text { Not Home Derm Dept }(24,3.92,4.00)\end{array}$ & $\begin{array}{l}\text { No concern }(7,1) \\
\text { Slightly }(11,2) \\
\text { Somewhat }(11,3) \\
\text { Moderately }(11,10) \\
\text { Extremely }(5,8)\end{array}$ & $0.00^{t}$ & \\
\hline \multirow[t]{2}{*}{$\begin{array}{l}\text { How concerned are you about getting an } \\
\text { accurate sense of program culture via } \\
\text { virtual interviews? }\end{array}$} & $\begin{array}{l}\text { UIM }(34,4.56,5.00) € \\
\text { Non-UIM }(33,3.94,4.00)\end{array}$ & $\begin{array}{l}\text { No concern }(0,0) \\
\text { Slightly }(0,3) \\
\text { Somewhat }(2,5) \\
\text { Moderately }(11,16) \\
\text { Extremely }(21,9)\end{array}$ & $0.00^{\ddagger}$ & \\
\hline & $\begin{array}{l}\text { UIM }(34,3.18,3.00)^{€} \\
\text { Non-UIM }(33,2.45,2.00)\end{array}$ & $\begin{array}{l}\text { No concern }(4,11) \\
\text { Slightly }(10,8)\end{array}$ & $0.04^{t}$ & \\
\hline
\end{tabular}

July 2021 Volume 5 Issue 4 


\section{SKIN}

\begin{tabular}{|c|c|c|c|c|}
\hline \multirow[t]{3}{*}{$\begin{array}{l}\text { How concerned are you about acquiring } \\
\text { dermatology specific letters of } \\
\text { recommendation? }\end{array}$} & & $\begin{array}{l}\text { Somewhat }(7,6) \\
\text { Moderately }(2,4) \\
\text { Extremely }(11,4)\end{array}$ & & \multirow[b]{2}{*}{ MS3-MS4 $(0.01)^{ \pm}$} \\
\hline & $\begin{array}{l}\text { MS3 }(14,3.71,4.00) \\
\text { MS4 }(50,2.58,2.00) \\
\text { Other }(5,3.20,3.00)\end{array}$ & $\begin{array}{l}\text { No concern }(1,14,0) \\
\text { Slightly }(2,14,2) \\
\text { Somewhat }(4,8,1) \\
\text { Moderately }(0,7,1) \\
\text { Extremely }(7,7,1)\end{array}$ & $0.03^{1}$ & \\
\hline & $\begin{array}{l}\text { Home Dern Dept }(45,2.47,2.00) \\
\text { Not Home Derm Dept }(24,3.58,4.00)\end{array}$ & $\begin{array}{l}\text { No concern }(13,2) \\
\text { Slightly }(14,4) \\
\text { Somewhat }(8,5) \\
\text { Moderately }(4,4) \\
\text { Extremely }(6,9)\end{array}$ & $0.00^{t}$ & \\
\hline $\begin{array}{l}\text { How concerned are you that a virtual } \\
\text { visiting dermatology elective may not } \\
\text { afford adequate interaction for a strong } \\
\text { letter of recommendation? }\end{array}$ & $\begin{array}{l}\text { Home Derm Dept }(45,3.53,4.00) \\
\text { Not Home Derm Dept }(24,4.33,5.00)\end{array}$ & $\begin{array}{l}\text { No concern }(6,1) \\
\text { Slightly }(4,1) \\
\text { Somewhat }(8,2) \\
\text { Moderately }(14,5) \\
\text { Extremely }(13,15)\end{array}$ & $0.01^{ \pm}$ & \\
\hline $\begin{array}{l}\text { How concerned are you about not having } \\
\text { any in-person dermatological conferences } \\
\text { or networking events? }\end{array}$ & $\begin{array}{l}\text { UIM }(34,4.24,4.00)^{€} \\
\text { Non-UIM }(33,3.64,4.00)\end{array}$ & $\begin{array}{l}\text { No concern }(0,3) \\
\text { Slightly }(2,2) \\
\text { Somewhat }(4,9) \\
\text { Moderately }(12,9) \\
\text { Extremely }(16,10)\end{array}$ & $0.04^{t}$ & \\
\hline $\begin{array}{l}\text { Would you be concerned about not being } \\
\text { able to interact with other peers } \\
\text { interviewing for the same programs as } \\
\text { you would have the opportunity during in } \\
\text { person interviews? }\end{array}$ & $\begin{array}{l}\text { UIM }(34,3.59,4.00)^{€} \\
\text { Non-UIM }(33,2.91,3.00)\end{array}$ & $\begin{array}{l}\text { No concern }(6,2) \\
\text { Slightly }(1,10) \\
\text { Somewhat }(5,12) \\
\text { Moderately }(11,7) \\
\text { Extremely }(11,2)\end{array}$ & $0.01^{ \pm}$ & \\
\hline $\begin{array}{l}\text { Are you concerned about not having a } \\
\text { home dermatology department? }\end{array}$ & $\begin{array}{l}\text { Home Derm Dept }(45, n / a, n / a) \\
\text { Not Home Derm Dept }(24, n / a, n / a)\end{array}$ & $\begin{array}{l}\text { Yes }(1,24) \\
\text { No }(4,0) \\
\text { N/A }(40,0)\end{array}$ & $0.000^{\mathrm{a}}$ & \\
\hline $\begin{array}{l}\text { Are you concerned about the financial } \\
\text { burden associated with test cancellations, } \\
\text { testing in different states or, testing } \\
\text { educational subscription extensions? }\end{array}$ & $\begin{array}{l}\text { MS3 }(14, n / a, n / a) \\
\text { MS4 }(50, n / a, n / a) \\
\text { Other }(5, n / a, n / a)\end{array}$ & $\begin{array}{l}\text { Yes }(14,16,4) \\
\text { No }(0,23,0) \\
\text { N/A }(0,11,1)\end{array}$ & $0.000^{a}$ & \\
\hline
\end{tabular}




\section{SKIN}

\section{Which of the following impacts has COVID-19 had on your research projects?}

Home Derm Dept

$(45, n / a, n / a)$

Not Home Derm Dept $(24, n / a, n / a)$
Cancelled/stopped $(10,4)$

Delayed $(20,19)$

No significant changes $(14,1)$

Expedited completion $(1,0)$

‡ Mann Whitney Nonparametric test for difference of medians (two independent populations)

+ Kruskal-Wallis test for difference of medians (more than two independent populations)

a Chi Square Test for Independence

$€$ Note: Two respondents were excluded from the race analysis because they did not specify their specific race/ethnicity.

$\mathrm{n}=$ \# observations, mean=Likert mean, median=Likert media

Table 2. Respondent Demographics Stratified by Medical School Class

\begin{tabular}{|c|c|c|c|c|}
\hline & MS3 (n=14) & $\begin{array}{c}\text { MS4 } \\
(n=50)\end{array}$ & Other $(n=5)$ * & Total $(n=69)$ \\
\hline \multicolumn{5}{|l|}{ Gender } \\
\hline Female & 11 & 35 & 3 & 49 \\
\hline Male & 3 & 15 & 2 & 20 \\
\hline Nonbinary & 0 & 0 & 0 & 0 \\
\hline Prefer Not to Answer & 0 & 0 & 0 & 0 \\
\hline $\begin{array}{c}\text { Native American or Alaskan } \\
\text { American }\end{array}$ & 0 & 0 & 0 & 0 \\
\hline Asian or Pacific Islander & 2 & 9 & 1 & 12 \\
\hline Black & 8 & 10 & 0 & 18 \\
\hline White & 1 & 14 & 3 & 18 \\
\hline Hispanic or Latinx & 2 & 11 & 1 & 14 \\
\hline Other $t$ & 1 & 6 & 0 & 7 \\
\hline Northeast & 4 & 11 & 2 & 17 \\
\hline South & 4 & 15 & 0 & 19 \\
\hline West & 1 & 2 & 1 & 4 \\
\hline Midwest & 5 & 22 & 2 & 29 \\
\hline
\end{tabular}

Abbreviations and Symbols: MS3, third-year medical students; MS4, fourth-year medical students.

*Students indicated the following, Research fellowship, Masters programs, and $5^{\text {th }}$ year medical student.

$\dagger$ Students Race or Ethnicity included: Nigerian American, South Asian, Arab American, Middle Eastern, two of the above, and Black and White. 


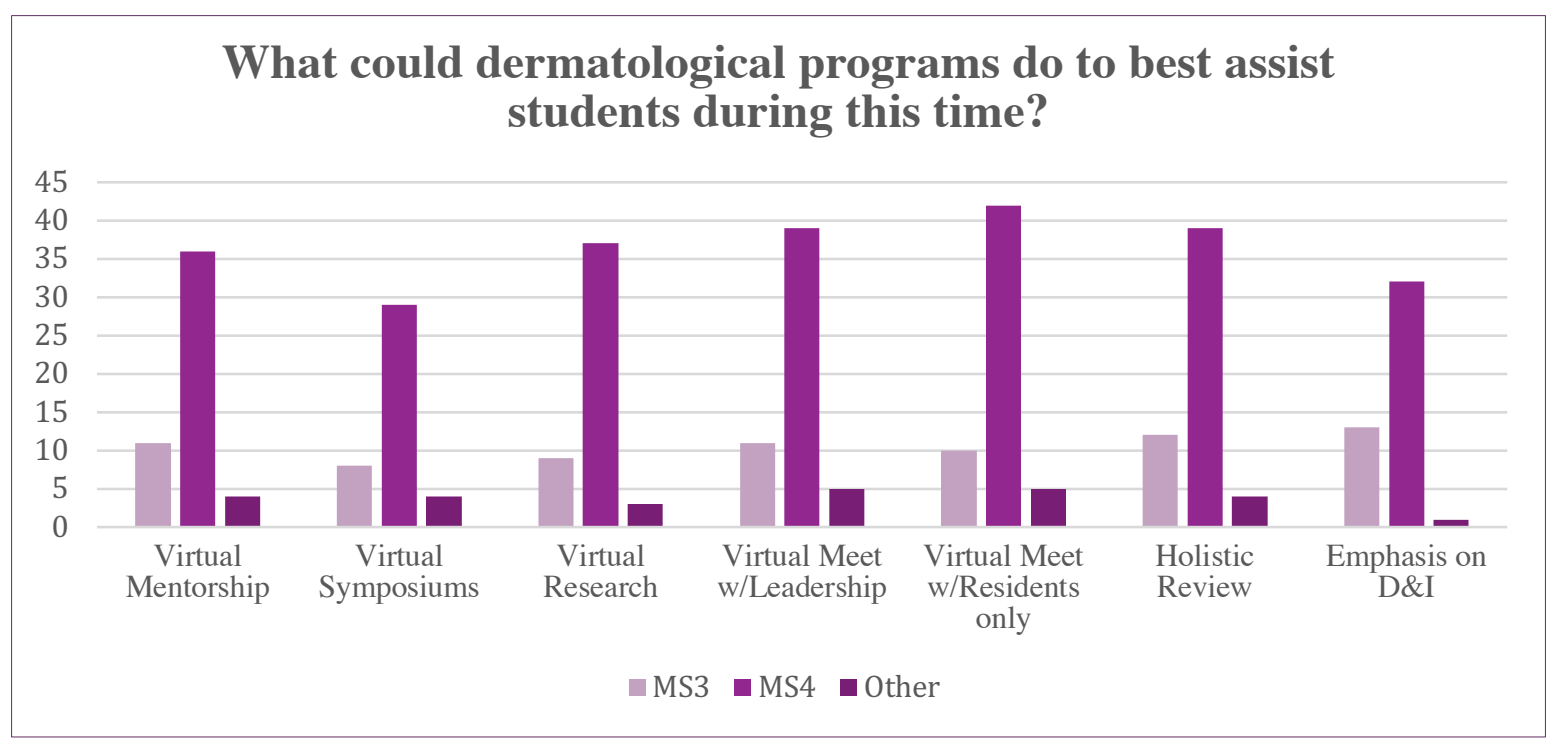

Figure 1. Suggestions Made by Medical Students for Virtual Residency Cycle Support

\section{Conflict of Interest Disclosures: None}

Funding: None

\section{Corresponding Author:}

Maria Tsoukas, MD, PhD

University of Illinois Hospital \& Health Sciences

$808 \mathrm{~S}$. Wood St,

Chicago, Illinois 60612

Phone: 312- 413-7767

Email: tsoukasm@uic.edu
5. National Resident Matching Program. Results and Data: 2020 Main Residency Match.

https://www.nrmp.org/main-residency-match-data/. Published 2020. Accessed March 26, 2021.

\section{References:}

1. Rosman IS, Schadt CR, Samimi SS, Rosenbach

$\mathrm{M}$. Approaching the dermatology residency application process during a pandemic. J Am Acad Dermatol. 2020.

2. Dermatology Residency Program Director Consensus Statement and Recommendations Regarding the 2020-2021 Application Cycle. https://studentsresidents.aamc.org/media/9526/download. Published 2020. Accessed April 12, 2021.

3. Association of the American Medical Colleges. ERAS Statistics. https://www.aamc.org/datareports/interactive-data/eras-statistics-data. Published 2020. Accessed March 26, 2021.

4. National Resident Matching Program. 2021 Main Residency Match Results. https://www.nrmp.org/main-residency-match-data/. Published 2021. Accessed March 26, 2021. 